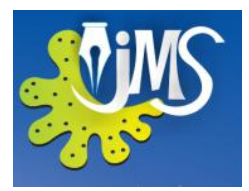

\title{
Propensity of Knowledge Sharing in Software Development: Case in Sri Lanka
}

\author{
Sudheera Weerakoon $^{1}$ and Janaka Wijayanayake ${ }^{2}$ \\ ${ }^{1}$ Paragon Software Lanka (Pvt.) LTD, Sri Lanka \\ ${ }^{2}$ Department of Industrial Management, \\ University of Kelaniya, Sri Lanka
}

\begin{abstract}
Knowledge management can be defined as the process by which an organization creates captures, acquires, and uses knowledge to support and improve the performance of an organization. Organizations need to manage knowledge within the organization to successfully face challenges and to achieve organizational goals. However, for better implementation of knowledge management practices, organizations need to have suitable organizational culture, process and technology. Software development is a process where knowledge sharing is vital for successful completion of projects and hence need proper knowledge sharing. Therefore, the objective of this study is to find the motivational factors that affect propensity of knowledge sharing in software development. To find the motivational factors, based on the findings of research in the literature, a conceptual model was developed with four independent variables, namely "Perception on knowledge", "Time availability for knowledge sharing", "Gained value from knowledge" and "Distance with employees". The conceptual model was validated with data collected from software developers using a questionnaire and interviews. Data Analysis showed that perceptions on knowledge and time availability for knowledge sharing have positive moderate correlation with propensity of knowledge sharing. However, it was found that distance with employees has negative weak correlation with propensity of knowledge sharing in software development organizations. Further, it was found that gained value from knowledge has negative weak correlation with propensity of knowledge sharing indicating that if employees get more and more benefits from the knowledge they have, then they are reluctant to share the knowledge with others.
\end{abstract}

KEYWORDS : Knowledge management, Knowledge Sharing, Software Development 


\section{INTRODUCTION}

Knowledge management can be defined as the process by which an organization creates captures, acquires, and uses knowledge to support and improve the performance of organizations. Organizations need to manage knowledge within the organization to successfully face challenges and to achieve organizational goals. By using knowledge management tools, organizations can capture and disseminate valuable knowledge resources among employees. However, for better implementation of knowledge management practices, organizations need to have suitable organizational culture, process and technology. Software development is a process where knowledge sharing is vital for successful completion of projects. Software projects that are in various domains are handled by software development companies and each and every project needs specialty knowledge to complete them successfully. By working on different projects, employees acquire unique knowledge with years of their experience. However, if knowledge sharing is not happening within an organization, when employees leave the organization, the team members of software development projects find it difficult to continue their projects. Therefore, having a proper mechanism to share knowledge is a very important aspect in software development. Hence, the objective of this study is to find the motivational factors that affect the propensity of knowledge sharing in software development.

\section{CONCEPTUAL MODEL}

After a thorough review of the available literature, four factors, namely, perception of knowledge, time availability for knowledge sharing, gained value from knowledge, and distance between employees have been identified as the main factors that affect knowledge sharing. Perception of knowledge is considered as one of the main factors for knowledge transfers. According to Herrgard (Augier, M. and Vendelo, M.T. (EDS) 1999). "Perception and language are considered the main difficulties in sharing tacit knowledge". Since tacit knowledge is internal understanding, perception of knowledge should be strong; otherwise the knowledge will not be shared properly. According to Nonaka's (Chun W.C and Sylvio C 2010). knowledge transfer model, internalization needs more time to establish knowledge in the requesters' mind and therefore, time factor affects the sharing of knowledge among workers. Further, internalization of knowledge requires a longer time not only within individual, even organizational forms of knowledge require longer time for internalization (Herrgard, T.H 2000). Therefore, time availability is a factor that affects knowledge sharing. Knowledge has a value. Knowledge helps to gain power and knowledgeable employees get receive benefits from organizations. This situation creates negative influence on knowledge sharing. According to Herrgard (Augier, M. and Vendelo, M.T. (EDS) 1999). value of knowledge is a vital factor that influences knowledge sharing. According to literature, distance can be categorized into "Functional Distance", "Power Distance" and "Physical Distance" and these factors are some of the main factors that affect knowledge sharing (Nonaka, I. and takeuchi, H. 1995). Knowledge transformation required face to face communication, inspections and human interactions for successful knowledge transfer. Therefore, distance is a main factor that affects knowledge transfer. 


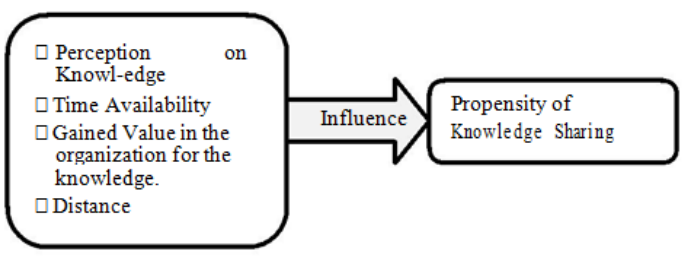

Figure 1: Factors affecting propensity of knowledge sharing

\section{DATA COLLECTION AND RESULTS}

In order to test the correlation between factors identified and the propensity of knowledge sharing, for each factor, measurable variables were selected. Derivation of variables was based on well known books, articles and research papers. A questionnaire was used as the data collection tool for the survey. For this, the variables identified for each factor were mapped into questions to be used in the questionnaire. 46 questioners were distributed among selected group of employees who play different roles in software development. All 46 were interviewed with the questionnaire to collect data accurately. As data collected were non-parametric, Spearman's Correlation was used to find the correlation between factors in the conceptual model. The resulted correlation coefficients for the factors are shown in Table 01

Table 1: Spearman Correlation Coefficient

\begin{tabular}{lll}
\hline Factors & Correlation Coefficient & Relationship \\
\hline Perception On Knowledge & 0.536 & Positive Moderate \\
Time Availability & 0.629 & Positive Moderate \\
Gained Value for the & -0.420 & Negative Weak \\
knowledge & -0.396 & Negative Weak \\
Distance & &
\end{tabular}

According to the analysis of data with SPSS Version 17, perception on knowledge and time availability have a positive moderate correlation with the propensity of knowledge sharing with co-efficient value of 0.536 and 0.629 respectively. However, gained value for knowledge and distance between employees have a weak negative correlation with the propensity of knowledge sharing with coefficient value of -0.420 and -0.396 respectively.

According to the results, it is proven that perception on knowledge is having a positive moderate influence with the propensity of knowledge sharing. When analyzing answers to questions, it was found that $72 \%$ of employees indicated that they have sufficient knowledge about theoretical background, proper knowledge about systematic procedures and self-awareness about their knowledge, and they answered that they have a good perception of knowledge. Around 30\% of employees have no clear perception of theoretical background and perception of systematic procedures, but a majority of employees were confident about their knowledge and skills. Even though the perception of knowledge has a moderate correlation with knowledge sharing, since perception of knowledge among employees is strong, this factor may not be a main factor that affects knowledge sharing in software development.

Data analysis proven that time availability has a positive moderate influence with the propensity of knowledge sharing. This describes that when available free time increases, propensity of knowledge sharing also increases. But when analyzing answers given for the available free time, only $37 \%$ answered directly that they are willing to share their knowledge in their free time. In addition a $29 \%$ of employees answered that they have a fair amount of free time during office hours and nearly $70 \%$ of employees answered that low or no free time is available during office hours. Since it gives a positive relationship, organizing a knowledge sharing times during office hours 
may be a method that could be used to improve knowledge sharing in software development.

According to the research findings, it is proven that gained value for knowledge has a negative moderate influence with the propensity of knowledge sharing. This finding indicates that if employees get more and more benefits from the knowledge they have, then they are reluctant to share the knowledge with others. This finding confirms the findings of Herrgard (Augier, M. and vendelo, M.T. (EDS) 1999). where he found that employee are reluctant to share knowledge in order to keep their power. However, this research did not measure the reasons for the low intention of knowledge sharing due to gained value. Hence, further research is needed to find out the reasons.

Results also indicated that knowledge sharing habit or propensity of sharing will increase when requester is in the same team, and requestor is having the same power and requestor is in the same premises. Further, nearly $71 \%$ of employees willing to share knowledge with own team members and reluctant to share with employees who are in other teams, or employees who are with different power levels and employees who are in different locations. Therefore, employees need to be rotated into different teams and that will bring a close working environment among employees which may lead to improved knowledge sharing.

\section{CONCLUSION}

Perception on knowledge, time availability, gained value for knowledge and distance of employees were identified as factors that affect the propensity of knowledge sharing with moderate and weak correlation. Among those four factors, gain value for the knowledge factor and distance of employees showed negative weak relationship with the propensity of knowledge sharing, and others made positive moderate relationship with the propensity of knowledge sharing. These conclusions suggest that implementing morning meetings in agile software development mechanism to eliminate time availability factors, and improving knowledge sharing through documentation process. In addition job rotation, team rotation, and project rotation also are suggested to avoid the distance factor.

\section{REFERENCES}

AUGIER, M. AND VENDELO, M.T. (EDS) (1999). Networks, cognition and management of tacit knowledge, Journal of Knowledge Management, 3(4), pp. 252-61

CHUN W.C. AND SYLVIO C. (2010). The individual and social dynamics of knowledge sharing: an exploratory study, Emerald Group Publishing Limited, 66(6), pp. 825-843BRACEWELL, R.N. 1965. The Fourier Transform and its applications, McGraw Hill, London, 444 pp.

HERRGARD,T.H. (2000). Difficulties in diffusion of tacit knowledge in organizations, Journal of Intellectual Capital, 1 (4) pp. 357 - 365

NONAKA, I. AND TAKEUCHI, H. (1995). The Knowledge Creating Company, Oxford University Press, New York, NY. 